\title{
Medical Image of the Week: Bullous Emphysema
}

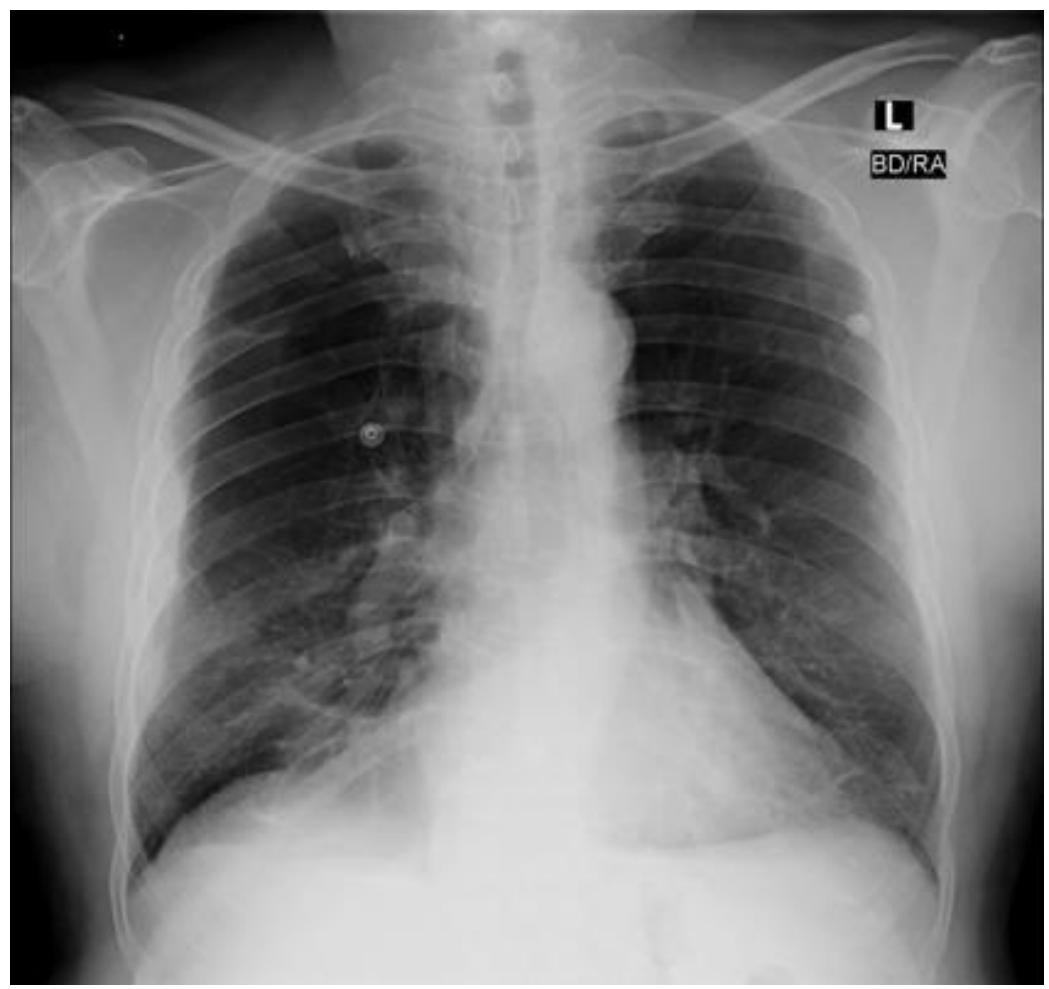

Figure 1. Chest radiograph showing hyperinflated lungs.
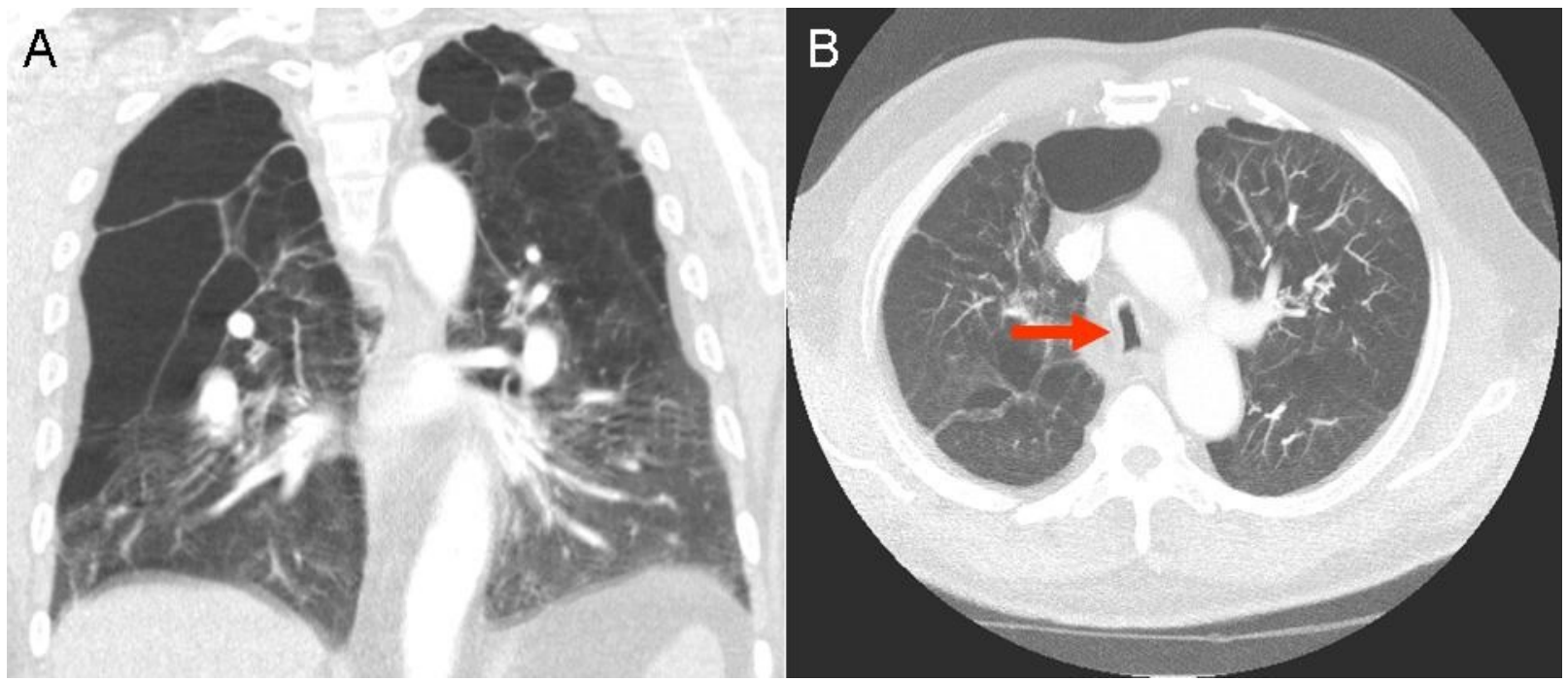

Figure 2. Panel A: Coronal view of chest computed tomography (CT) in lung widows showing multiple large lucent spaces of lung parenchyma destruction interspersed normal lung tissue. Panel B: Axial view of chest CT showing coronal narrowing of the trachea with widening of the sagittal diameter (arrow). This is known as a saber sheath trachea which is pathognomonic of chronic obstructive pulmonary disease. 
A 63-year-old gentleman, with a history of 90-pack-years of smoking and stage IV chronic obstructive pulmonary disease was receiving home oxygen at $2 \mathrm{~L} / \mathrm{min}$ at baseline. He has had multiple prior hospital admissions for respiratory failure. Over the past 2 weeks he has had increased production of sputum, associated with worsening shortness of breath. He is on fluticasone-salmeterol inhaler, albuterol inhaler, and tiotropium as an outpatient.

On examination, he was hemodynamically stable, $\mathrm{SpO} 2$ was $92 \%$ on $4 \mathrm{~L} / \mathrm{min}$ of oxygen. He was in obvious respiratory distress, in a tripod position with tachypnea and using respiratory accessory muscles. Lung examination revealed diffuse expiratory wheezing.

Chest radiograph shows severe emphysema (Figure 1 ). Chest computed tomography showed diffuse centrilobular and bullous emphysema (Figure 2). He was treated as an acute severe exacerbation of COPD and was eventually discharged to follow-up with the pulmonary clinic.

Emphysema is defined as alveolar destruction and airspace enlargement distal to the terminal bronchiole. There are subclassifications of emphysema based on its distribution within the secondary lobule (1). Bullae are defined as an air-filled space, greater than $1 \mathrm{~cm}$ in diameter, usually as a result of emphysematous destruction. Indications for bullectomy include patient symptoms, isolated bullae occupying $>30 \%$ of the hemithorax or complications arising from bullae (2).

Kai Rou Tey, MD¹; Akinbola Ajayi-Obe ${ }^{1}, \mathrm{MD}$; and Naser Mahmoud, MD ${ }^{2}$

${ }^{1}$ Department of Internal Medicine, South Campus

${ }^{2}$ Department of Pulmonary, Critical Care, Allergy and Sleep

University of Arizona College of Medicine

Tucson, AZ

\section{References}

Terminology, Definitions, and Classification of Chronic Pulmonary Emphysema and Related Conditions: A Report of the Conclusions of a Ciba Guest Symposium. Thorax. 1959;14(4):286-299.

van Berkel V, Kuo E, Meyers BF. Pneumothorax, bullous disease, and emphysema. Surg Clin North Am. 2010 Oct;90(5):935-53. [CrossRef] [PubMed] 